\title{
Computation of the controllable swing mode spectrum of FACTS and HVDC in large power systems
}

\author{
VEDANTA PRADHAN*(D, A M KULKARNI and S A KHAPARDE \\ Department of Electrical Engineering, Indian Institute of Technology Bombay, Mumbai 400 076, India \\ e-mail: vedanta.pradhan@gmail.com
}

MS received 28 October 2016; revised 10 July 2018; accepted 12 July 2018; published online 12 September 2018

\begin{abstract}
This paper presents computation of swing modes of a large power system that could be significantly affected by power swing damping controllers in FACTS or HVDC devices at a given location. Modal controllability is a suitable measure to isolate these modes for analysis. Computation of the controllable swing mode spectrum is useful, especially in situations where the controller structure and feedback signals are not frozen (e.g., at the planning stage). This paper proposes two important steps that allow us to map the problem of finding highly controllable swing modes to the problem of finding the swing modes that have high transfer function residues (for which efficient algorithms are available). The steps are: (a) normalization of the eigenvectors corresponding to different modes and (b) identification of specific feedback signals for each type of FACTS/ HVDC device such that the modal observability and modal controllability are tightly coupled. Once the mapping is done, a computationally efficient method like the Subspace Accelerated Dominant Pole Algorithm [16] (SADPA) can be adapted to find the highly controllable swing modes. The effectiveness of this approach is demonstrated by case studies of FACTS and HVDC devices in a 16-machine system and the Indian power grid.
\end{abstract}

Keyword. Power system dynamics; selective eigenvalue computation; FACTS; HVDC.

\section{Introduction}

FACTS and HVDC systems play a key role in stabilizing the electro-mechanical dynamics $[1,2]$ of power systems. Typically, a device at a given location exerts control on a few swing modes among the very many that exist in a large power system. An important question that arises during dynamic analysis and eigenvalue computation is the following: which are the swing modes that are controllable by the device? Knowledge of the modal controllabilities ensures that all eigenvalues that potentially could get affected by the controller can be tracked. If the controller and feedback signals are known, this could be answered by carrying out an eigenvalue analysis to track all modes that move with increasing controller gain. In fact residue-based algorithms [3] can be used to selectively compute such modes. This is however not feasible if the feedback signal and controller structure are not frozen (as in the planning stage). Moreover, with the advent of Wide Area Measurement Systems (WAMS) there is a wider choice of feedback signals that may be used. Under these circumstances, modal controllability is the most appropriate measure. It characterizes the inherent ability of the device to control a mode without reference to a specific controller or feedback signal.

*For correspondence
While modal controllability (and the set of highly controllable swing modes) can be obtained from the complete eigen-solution, it is not feasible to compute all eigenvalues for practical systems due to the large number of states. Therefore, selective eigenvalue analysis algorithms catering to different requirements have been developed as shown in figure 1 . They include selective computation of unstable eigenvalues [4], eigenvalues that participate highly in the states of a small study zone of the system [5], eigenvalues in specified regions of the complex plane [6] and weakly damped modes [7-9]. Algorithms proposed in [10-12] are suitable for finding the sensitive eigenvalues with respect to changes of control and other system parameters like loading and network parameters. In $[13,14]$, the swing modes associated with a particular generator are identified from the dominant poles in the transfer function between its rotor slip and prime-mover input. Generalized algorithms are reported in $[15,16]$ to find the dominant poles of any given single-input singleoutput (SISO) transfer function.

Dominant pole algorithms may miss out on some highly controllable modes if the output signal has poor observability of the modes. This motivates us to devise a selective eigen-analysis tool that can directly compute all the highly controllable swing modes for a given device location. A difficulty in developing such a method is that modal controllability across different modes cannot be compared 
Vedanta Pradhan et al

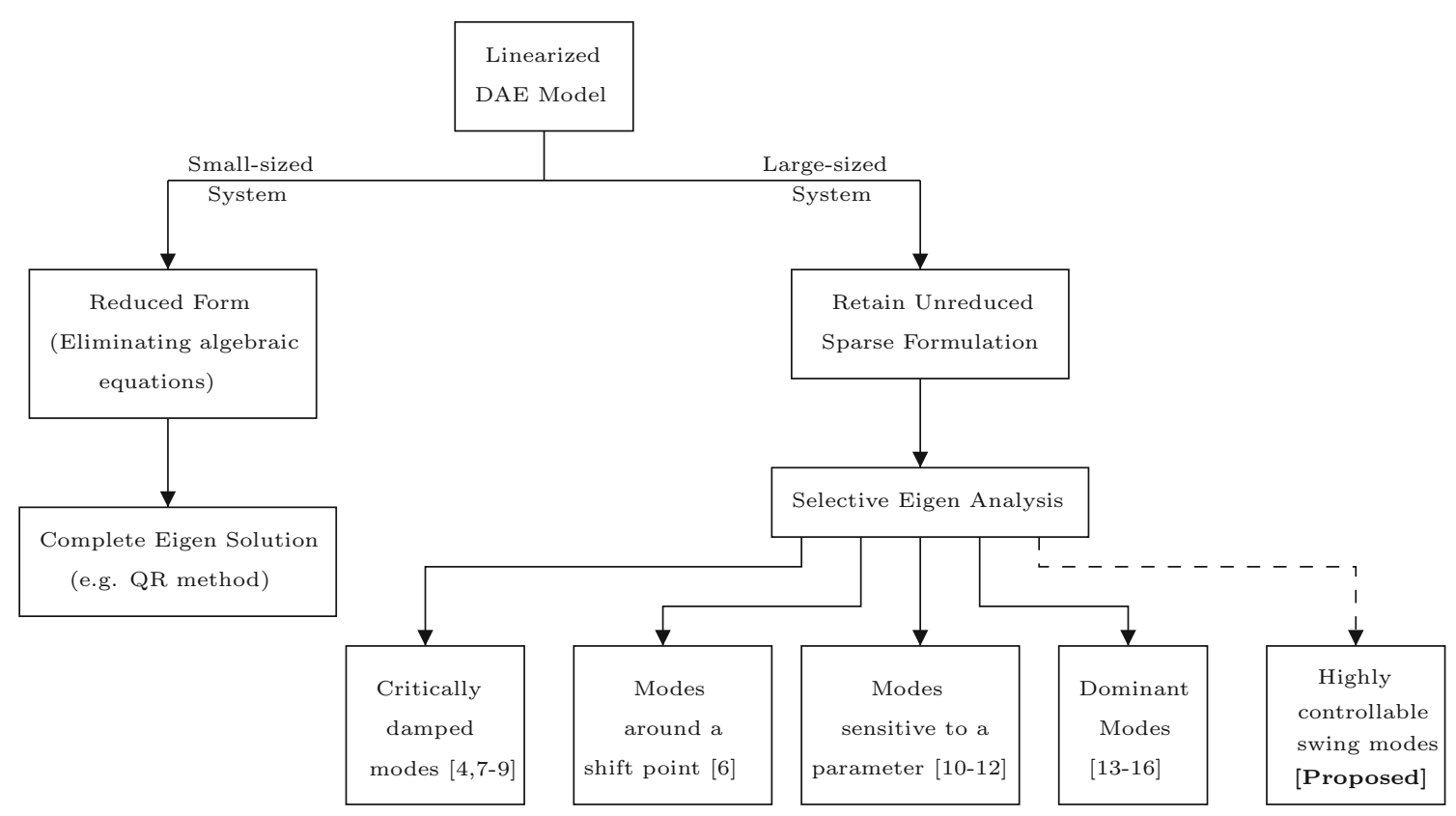

Figure 1. Computational tools for selective eigen-analysis.

unless there are constraints on the scaling of the corresponding eigenvectors. The paper aims to approach this by mapping the problem of finding the highly controllable modes to that of computing the modes that have high transfer function residues. The main contribution of the paper is in proposing two important steps that allow this mapping. They are the following.

(a) Normalization of the eigenvectors corresponding to different swing modes in a way that allows a fair comparison of modal controllabilities. If one mode is excited at a time, disturbance energy being the same, the normalization proposed in this paper ensures that each mode has equal usage of the device range, if the corresponding modal signal is used as a feedback signal.

(b) Identification of specific feedback signals for each type of FACTS/HVDC device such that highly controllable swing modes (for a given device) are highly observable in the corresponding feedback signal and vice-versa, i.e., modal observability and modal controllability are tightly coupled.

Once this mapping is done, a suitable adaptation of an efficient residue-based algorithm like Subspace Accelerated Dominant Pole Algorithm (SADPA) [16] is used to formulate an iterative computational method. This is demonstrated by case studies using a 16-machine 68-bus system and the Indian power grid.

Note that the aim of the paper is not to propose feedback signals or means to evaluate feedback signals for damping control design. The choice of specific feedback signals is only to facilitate the mapping of the modal controllabilities to residues. This shall become clearer in section 2 .

\section{Modal controllability of swing modes}

This section aims to analyse the modal characteristics of a simplified power system model in which generators are represented by the classical model, prime-mover inputs are held constant, line losses are ignored and real and reactive parts of loads are modelled as constant power and impedance, respectively. The aim of the analysis is to (a) propose a reasonable normalization of eigenvectors for fair comparison of modal controllabilities among swing modes and (b) identify special feedback signals for each type of FACTS/HVDC device that allow us to use efficient residuebased algorithms like SADPA.

\subsection{Modal analysis of a power system}

Let us consider the SISO state-space model of the power system with $u$ and $y$ as the input and output variables, respectively, as shown here:

$$
\begin{aligned}
& \dot{z}=A z+b u, \\
& y=c z+d u .
\end{aligned}
$$

For the simplified model of the system the state variable vector $z$ consists of machine rotor angles $\delta$ and speeds $\omega$, 
i.e., $z=\left[\Delta \delta^{T} \Delta \omega^{T}\right]^{T}$. The system matrix has the following form [17]:

$$
A=\left[\begin{array}{cc}
0 & I \\
-M^{-1} A_{r} & 0
\end{array}\right]
$$

$M$ is an $n_{g} \times n_{g}$ diagonal matrix with the $m$ th diagonal entry equal to $2 H_{m} / \omega_{B}$, where $H$ stands for generator inertia constant and $\omega_{B}$ is machine base speed. A few important observations can be made based on the special structure of the model, which are listed here. Details of these derivations and inferences can be found in [17].

1. $A_{r}$ is symmetric.

2. The system matrix $A$ has $\left(n_{g}-1\right)$ pairs of complexconjugate eigenvalue pairs $\left( \pm j \Omega_{l}, l=1\right.$ to $\left.n_{g}-1\right)$ that correspond to the "swing modes" and two zero eigenvalues.

3. Let $x_{i}=\left[\begin{array}{c}x_{\delta_{i}} \\ x_{\omega_{i}}\end{array}\right]$ be the right eigenvector corresponding to the eigenvalue $\lambda_{i}=j \Omega_{i}$. From the definition of eigenvectors $\left(A x_{i}=\lambda_{i} x_{i}\right)$, we have the following relationship:

$$
\begin{aligned}
x_{\omega_{i}} & =\lambda_{i} x_{\delta_{i}} \text { and }-M^{-1} A_{r} x_{\delta_{i}}=\lambda_{i} x_{\omega_{i}} \\
\Rightarrow A_{r} x_{\delta_{i}} & =-\lambda_{i}^{2} M x_{\delta_{i}} .
\end{aligned}
$$

4. The left eigenvector $v_{i}=\left[\begin{array}{c}v_{\delta_{i}} \\ v_{\omega_{i}}\end{array}\right]$ is related to the right eigenvector $x_{i}$ as follows:

$$
\left[\begin{array}{c}
v_{\delta_{i}} \\
v_{\omega_{i}}
\end{array}\right]=h_{i}\left[\begin{array}{c}
\lambda_{i} M x_{\delta_{i}} \\
M x_{\delta_{i}}
\end{array}\right]=h_{i}\left[\begin{array}{c}
M x_{\omega_{i}} \\
M x_{\delta_{i}}
\end{array}\right]
$$

where $h_{i}$ is an arbitrary scalar.

5. $v_{\omega_{i}}$ and $x_{\delta_{i}}$ can be chosen to be real. For the rest of the discussion in the paper, we assume $x_{\delta_{i}}$ to be real.

Assuming that the eigenvalues corresponding to the swing modes are distinct, we can decompose the system into $\left(n_{g}-1\right)$ modal systems by the following transformation of states:

$$
\begin{aligned}
{\left[\begin{array}{c}
\Delta \delta \\
\Delta \omega
\end{array}\right] } & =\sum_{i=1}^{n_{g}-1} x_{i} z_{m_{i}}+x_{i}^{*} z_{m_{i}}^{*} \\
& =\sum_{i=1}^{n_{g}-1}\left[\begin{array}{c}
x_{\delta_{i}} \\
x_{\omega_{i}}
\end{array}\right] z_{m_{i}}+\left[\begin{array}{c}
x_{\delta_{i}}^{*} \\
x_{\omega_{i}}^{*}
\end{array}\right] z_{m_{i}}^{*}
\end{aligned}
$$

$z_{m_{i}}=v_{i}^{T}\left[\begin{array}{c}\Delta \delta \\ \Delta \omega\end{array}\right]$ and $z_{m_{i}}^{*}=v_{i}^{H}\left[\begin{array}{c}\Delta \delta \\ \Delta \omega\end{array}\right]$ are the modal states corresponding to the $i$ th mode and the following normalization of eigenvectors holds:

$$
v_{i}^{T} x_{i}=1, v_{i}^{H} x_{i}^{*}=1 \quad \forall i .
$$

Using Eqs. (3) and (4) it can be shown that $h_{i}$ takes the following value to satisfy Eq. (6):

$$
h_{i}=\frac{-\lambda_{i}}{x_{\delta_{i}}^{T} A_{r} x_{\delta_{i}}-x_{\omega_{i}}^{T} M x_{\omega_{i}}} \quad \forall i .
$$

The $i$ th modal system is given by

$$
\begin{aligned}
& \dot{z}_{m_{i}}=\lambda_{i} z_{m_{i}}+v_{i}^{T} b u, \\
& \dot{z}_{m_{i}}^{*}=\lambda_{i}^{*} z_{m_{i}}^{*}+v_{i}^{H} b u .
\end{aligned}
$$

This transformation and modal decomposition assumes that the system dynamics are only due to the oscillatory (swing) modes. The rigid body mode (represented by the zero eigenvalue pair) is not excited.

Using the transformation of Eq. (5), the output $y$ is given by the following equation:

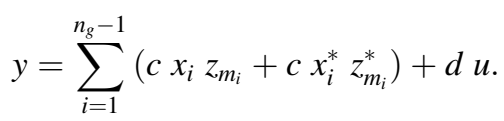

Modal controllability $\beta_{i}$ and modal observability $\gamma_{i}$ corresponding to $\lambda_{i}\left(=j \Omega_{i}\right)$ are defined as follows:

$$
\beta_{i}=v_{i}^{T} b, \quad \gamma_{i}=c x_{i}
$$

If a closed-loop feedback controller with the control law: $u=k y$ is used, the eigenvalue sensitivity $\frac{\mathrm{d} \lambda_{i}}{\mathrm{~d} k}$ as $k \rightarrow 0$ is the residue $\rho_{i}$ of the transfer function $\frac{y(s)}{u(s)}$ corresponding to $\lambda_{i}$, and is given by the following equation [18]:

$$
\rho_{i}=\left.\frac{\mathrm{d} \lambda_{i}}{\mathrm{~d} k}\right|_{k \rightarrow 0}=\frac{c x_{i} v_{i}^{T} b}{v_{i}^{T} x_{i}}=\gamma_{i} \beta_{i} .
$$

For a given output signal $y$, the relative effect of the controller on various eigenvalues can be compared using these sensitivities.

\subsection{Comparison of modal controllabilities of different modes}

Let us choose $y$ to be the modal signal corresponding to the $i$ th swing mode, i.e., $y=z_{m_{i}}=v_{i}^{T} z$ (i.e., $c=v_{i}^{T}$ and $d=0$ ). For this signal $\gamma_{i}=1$ and therefore $\rho_{i}=\beta_{i}$, i.e., if the modal signal is chosen as the feedback signal, then the corresponding residue highlights the modal controllability. However, comparison of modal controllabilities across different modes requires normalization of $v_{i}$, which is explained in the following paragraph.

A reasonable normalization criterion can be obtained if we ensure that "strength" of the modal signal $\left(z_{m_{i}}\right)$ for each mode is the same (assuming that only one mode is excited at a time and the modal disturbance energy is the same in each case). This in turn ensures that each mode has an equal opportunity to utilize the device control range (if the corresponding modal signal is used as a feedback signal, and 
Table 1. Dual pairs of FACTS/HVDC controllers.

\begin{tabular}{lcc}
\hline Control variable $u$ & Local dual signal $y_{d}$ & FACTS/HVDC device description \\
\hline$P_{s h}:$ Shunt-injected real power & $\phi:$ Bus voltage phase angle & DC converter terminal, STATCOM with energy \\
source. & STATCOM, SVC \\
$I_{R}:$ Shunt current in quadrature with bus & $V:$ Bus voltage magnitude & SSSC with energy source \\
voltage & $\zeta:$ Line current phase angle & TCSC, SSSC \\
$P_{s e r}:$ Series real power injection & $I:$ Line current magnitude & DC link \\
$V_{s e r}:$ Series voltage in quadrature with line & $\phi_{d}:$ Bus voltage phase angular \\
$P_{d c}:$ DC power flow & difference & \\
\hline
\end{tabular}

the control law is $u=\kappa z_{m_{i}}$, where $\kappa$ is feedback gain). The normalization criterion yields the following condition:

$$
x_{\delta_{i}}^{T} A_{r} x_{\delta_{i}}-x_{\omega_{i}}^{T} M x_{\omega_{i}}=1 \quad \forall i .
$$

The derivation is given in Appendix I. Equations (6), (7) and (12) completely define the normalization on the right and left eigenvectors.

Once the normalization is done, the residues for different swing modes obtained using the corresponding modal feedback signals can be compared to infer relative modal controllabilities. However, since the eigenvectors are not known a priori, the modal signals cannot be directly used in eigenvalue computation algorithms. The next section presents alternative signals using which the relative modal controllability can be inferred from residues. Nevertheless, the normalization still holds from the point of view of defining and comparing modal controllabilities across different swing modes.

\subsection{Dual variables}

Table 1 shows the control variables $(u)$ pertaining to various series and shunt controllable elements and the corresponding "dual" variables $\left(y_{d}\right)$. It has been shown in [17] that for such input-output pairs

$$
\beta_{i}=h_{i} \gamma_{i}^{d}
$$

where $\gamma_{i}^{d}$ denotes modal observability in $y_{d}$. From Eqs. (7) and (12), $h_{i}=-\lambda_{i}$, and therefore, $\left|\beta_{i}\right|=\left|\lambda_{i}\right|\left|\gamma_{i}^{d}\right|$. This suggests that for a swing mode, modal controllability of $u$ and modal observability in $y_{d}$ are tightly coupled. Highly controllable swing modes exhibit high residues $\left|\rho_{i}^{d}\right|$ in the corresponding transfer function and the following relationship holds:

$$
\left|\rho_{i}^{d}\right|=\left|\gamma_{i}^{d}\right|\left|\beta_{i}\right|=\left|\beta_{i}\right|^{2} /\left|\lambda_{i}\right|
$$

Since swing mode frequencies lie in a narrow range $(0.2-$ $2 \mathrm{~Hz}$ ), Eq. (14) suggests that highly controllable swing modes will exhibit relatively higher residues in the transfer function between the dual input-output pair. Using dual

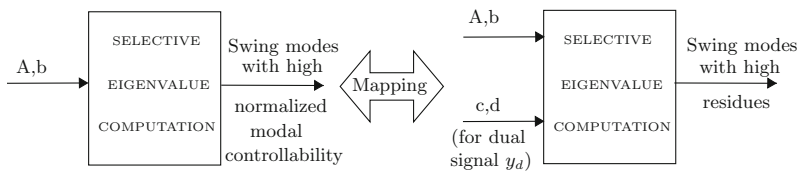

Figure 2. Mapping of the problem of finding the highly controllable swing modes to that of finding high-residue swing modes.

signal, the problem of finding the highly controllable swing modes is now mapped to that of finding modes with high $\left|\rho_{i}^{d}\right|\left|\lambda_{i}\right|$ as shown in figure 2 . Therefore, the dual signal is a suitable alternative to modal signals in facilitating the usage of residue-based algorithms for determining the highly controllable swing modes.

Although a simplified model of the system is assumed for the theoretical analysis, case studies shall use detailed models for practical demonstration. Moreover, practical implementation with large power systems shall use the sparse descriptor state-space formulation instead of the reduced (dense) state-space model [7]. We now present the proposed methodology, which uses the dual variable of a FACTS/HVDC device location for the computation of controllable swing modes.

\section{The proposed methodology}

The proposed methodology uses the dual variable for a given FACTS/HVDC location, adapts a residue-based algorithm, namely, SADPA and uses it as the core algorithm for eigenvalue computation. Before we present the details of the method, we first discuss the salient features of SADPA in brief.

\subsection{SADPA [16]}

Let us consider the SISO system (in descriptor form) for an input-output pair $(u-y)$ in general as follows:

$$
E \dot{z}=A z+b u, \quad y=c z .
$$


Its transfer function is given by the following equation:

$$
G(s)=\frac{Y(s)}{U(s)}=c(s E-A)^{-1} b=b^{T}(s E-A)^{-t} c^{T} .
$$

Poles with good observability and controllability (and hence high residues) can be described as dominant poles in the sense that the transfer function $G(s)$ can be effectively approximated by knowledge of the eigen-triplets (eigenvalue and eigenvectors) associated with such poles [3]. For a finite value of $U(s), G(s)$ tends to become unbounded at complex frequencies close to the dominant poles. Limiting $Y(s)$ to a finite value (say 1$), U(s) \rightarrow 0$, as $s$ approaches such a pole. This is the underlying principle of Dominant Pole Algorithm (DPA) [3]. With an initial estimate $s=s_{0}$, the algorithm seeks better estimates in an iterative manner. The estimate at the $p$ th iteration is given by the following equation:

$$
s_{p+1}=s_{p}-\frac{U\left(s_{p}\right)}{v\left(s_{p}\right)^{T} E x\left(s_{p}\right)} .
$$

This is derived from Generalized Rayleigh Quotient iteration, where $x\left(s_{p}\right)$ and $v\left(s_{p}\right)$ are, respectively, the right and left eigenvector estimates. The process converges as $s_{p}$ becomes equal to a dominant pole and $U\left(s_{p}\right) \rightarrow 0$. If $s_{p}$ is a pole that is either uncontrollable or unobservable, then $G\left(s_{p}\right) \rightarrow 0$ and $U\left(s_{p}\right) \rightarrow \infty ; s_{p+1}$ will be forced away from $s_{p}$.

While DPA can evaluate eigen-triplet corresponding to a single dominant pole at a time using a single initial estimate, SADPA [16] utilizes the concept of DPA in a subspace iteration-based algorithm to simultaneously evaluate multiple dominant poles. It has the following features.

Single initial estimate: The algorithm starts with a single initial pole estimate to compute the desired number of dominant poles. This is made possible by the subspace acceleration approach, rather than the single vector at a time approach followed in DPA.

Expansion of eigenspaces: Apart from evaluation of the next estimate as per Eq. (17), the information of approximate eigenvectors $x\left(s_{p}\right)$ and $v\left(s_{p}\right)$ of the $p$ th iteration is used to expand iterative eigenspaces $X$ and $V$. The expansion is performed by a Gram-Schmidt projection of the vectors onto the spaces $X$ and $V$. Therefore, it can also be said that the spaces are orthogonally expanded with the current iteration vectors. Such an expansion of the iterative eigenspaces seeks for diverse directions in the vector space and therefore accelerates the search. Expansion of these spaces allows for better approximations of multiple dominant poles.

Selection criterion: At the end of each iteration, the algorithm chooses the next best estimate out of the multiple approximate dominant poles by sorting them according to a criterion. For example, $\left|\rho_{i}\right| /\left|\operatorname{Re}\left(\lambda_{i}\right)\right|$ is used as the selection criterion in [16], for transfer function model order reduction.

Deflation: Once SADPA converges to a dominant pole, the iterative eigenspaces $X$ and $V$ are deflated by explicit skew projection of the vectors in the spaces with the converged left and right eigenvectors. The resulting spaces are then re-orthogonalized. This avoids repeated convergence in further iterations to the dominant poles already found.

Now we present details of the proposed methodology, which uses SADPA with a suitable selection criterion at its core for eigenvalue computation. The overall process is first presented, followed by discussion of selection and convergence criteria.

\subsection{Overall process}

For a device at any given location, the control variable $u$ and its corresponding dual signal $y_{d}$ as shown in table 1 are identified. The proposed method essentially involves a recursive application of SADPA to find eigen-triplets corresponding to the controllable swing modes. The method consists of an outer iterative loop whose function is to determine a finite number $(\hat{n})$ of controllable $\operatorname{modes}^{1}$ per iteration using SADPA. Note that this loop is called as "outer" in order to differentiate it from the iterative loop within SADPA itself. However, going forward, the term "outer" is not used as no reference is made to the iterations within SADPA henceforth.

The process may begin with an initial estimate (say $0+j 1$ ) of a controllable mode and null/empty eigen-subspace matrices $X$ and $V$. At the end of the $k$ th iteration, the best estimates of the iterative controllable pole and the iterative eigen-subspaces (at that point of time) are assigned to $s_{0}, X$ and $V$ for the $(k+1)$ th iteration. This iterative process may be stopped based on a suitable convergence criterion. The most important aspect of this procedure is the selection criterion to be used in SADPA. These aspects are discussed next. The overall procedure is summarized in flowchart form in figure 3.

3.2a Selection criterion: While $\left|\rho_{i}\right| /\left|\operatorname{Re}\left(\lambda_{i}\right)\right|$ is the default selection criterion in SADPA, it can be changed [16] to other indices of modal dominance. We modify it to meet our requirement. Equation (14) suggests the selection criterion to be $\left|\lambda_{i}\right|\left|\rho_{i}\right|$ for converging to the highly controllable modes. While it is feasible to use this criterion with the reduced state-space model, it is not so for the sparse descriptor state-space formulation. This is because a generalized eigenvalue problem with singular $E$ may have dominant poles at infinity [19]. Such poles have no physical significance and convergence of the algorithm towards infinity is computationally undesirable. Keeping the selection criterion as $\left|\rho_{i}\right|$ or $\left|\lambda_{i}\right|\left|\rho_{i}\right|$ drives the algorithm towards

${ }^{1}$ For complex poles, the complex-conjugate pair is considered as one mode. 


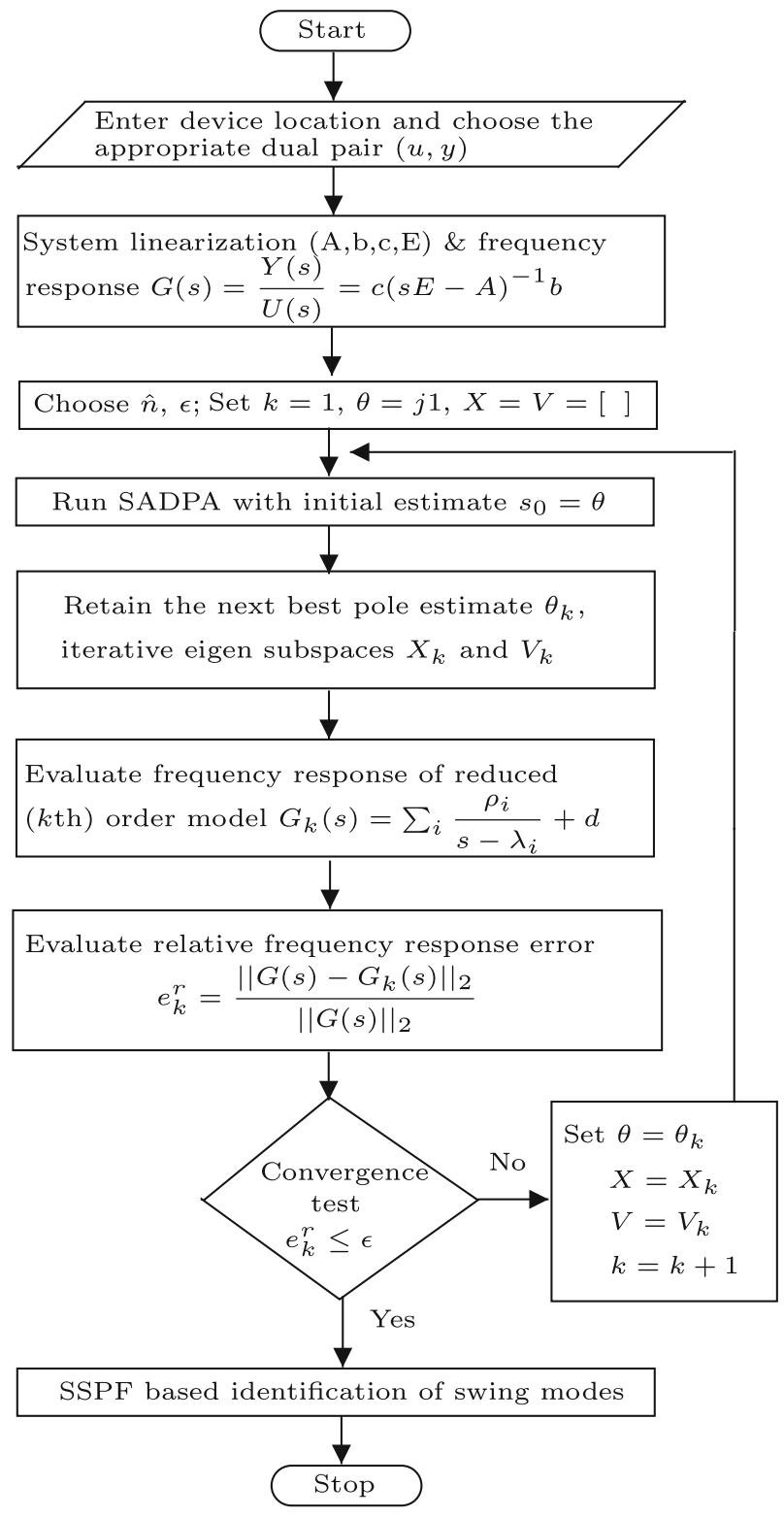

Figure 3. Process flow for the proposed methodology.

the infinite pole spectrum. In such a case, choosing the selection criterion as $\left|\rho_{i}\right| /\left|\lambda_{i}\right|$ is useful. This is demonstrated with the help of an example in Appendix II. However, in doing so, we are actually looking for poles with high $\left|\beta_{i}\right| /\left|\lambda_{i}\right|$ as is apparent from (14). However, this scheme is expected to converge to the controllable swing mode spectrum (with a slight bias towards the low frequency modes) since swing mode frequencies lie in a narrow range.

3.2b Convergence criterion: As the number of converged modes in a certain range of frequency increases, the reduced order transfer function (say after the $k$ th iteration) given by

$$
G_{k}(s)=\sum_{i=1}^{n p(k)} \frac{\rho_{i}}{s-\lambda_{i}}+d
$$

tends towards the actual frequency response $G(s)$ in that range of frequency [15]. The expression for $G(s)$ is given in Eq. (16). Of course, if a feed-forward term is present in the state-space representation of Eq. (15), then it has to be added to the expression given in (16). Note that $n p(k)$ is the number of dominant poles found after $k$ iterations and " $d$ " is the direct transmission element in the reduced state-space model of the system [12]. This process is allowed to continue till $G_{k}(s)$ approaches $G(s)$ to a convergence tolerance. The notion of Euclidean distance between the two is used to quantify relative error, which is given by

$$
e_{k}^{r}=\frac{\left\|G(s)-G_{k}(s)\right\|_{2}}{\|G(s)\|_{2}} .
$$

It is well known that high-residue poles that lie close to the imaginary axis are the most important for effective transfer function order reduction. Therefore, with this convergence criterion, the process not only captures the highly controllable swing modes but also continues till the modes that are relatively less controllable but lie close to the imaginary axis are also captured. The iterative procedure converges as $e_{k}^{r} \leq \epsilon$, where $\epsilon(0 \leq \epsilon<<1)$ is the transfer function convergence tolerance. A moderately low initial value (e.g., $10^{-2}$ ) can be assigned to it.

Closing of the computation loop via a convergence criterion makes the process automatic. Moreover, unnecessary computations are avoided as convergence implies that sufficient number of modes have been determined. The proposed approach can also be used in an open-loop manner when a very limited number of modes with the highest modal controllabilities are of interest. In the set of converged modes, sum of slip participation factors (SSPF) [20] can be used as an index to identify swing and non-swing modes.

\section{Results and discussion}

Case studies are done on the IEEE 16-machine 68-bus test system and the Indian power system. Detailed models are used for the demonstrations. The moderate size of the former facilitates a physical understanding and validation of the results obtained. The latter demonstrates the proposed method for a large practical system. The settings for SADPA [16] used in all the case studies are as follows: dimensions of minimum and maximum search spaces are 5 and 10, respectively; eigen-pair convergence tolerance is $10^{-10}$. Coding is done in MATLAB on an Intel(R) Core(TM) i5-4570 3.2 GHz CPU with 4 GB RAM.

\subsection{6-machine 68-bus NETS-NYPS system}

This system is divided into two areas, the NETS and the NYPS as shown in figure 4 . The external neighbouring systems are modelled by equivalent machines G14-G16. The base case power flow and dynamic data including the 


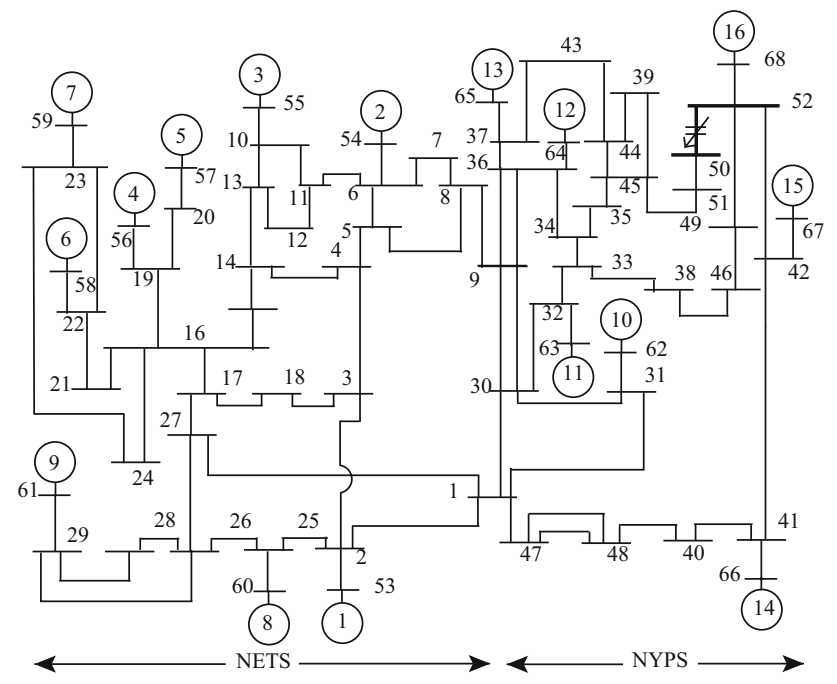

Figure 4. Sixteen-machine 68-bus single line diagram.

excitation systems and PSS data are adapted from [18]. Loads are modelled as having voltage and frequency dependence. For the computations, $\hat{n}$ is set to 2 .

4.1a TCSC: A TCSC is placed in the branch between buses 50 and 52 as shown in figure 4 . The series compensation is modelled as a variable voltage in quadrature with the line current. The controllable element is the magnitude of this voltage, i.e., $u=\Delta V_{\text {ser }}$. Hence the line (50-52) current magnitude is considered as the dual signal, i.e., $y_{d}=\Delta I$. Table 2 shows a partial list of the converged poles, their residues $\left(\left|\rho_{i}\right|\right)$ in the transfer function between the dual input-output pair and the modal controllabilities $\left|\beta_{i}\right|$ evaluated using Eq. (14). All the converged swing mode eigenvalues are listed in the table. Figure 5 shows the convergence over iterations (counted as $k$ ) and improvement in the match between the reduced order and the exact transfer functions $G(s)$ and $G_{k}(s)$, respectively. The plots in dash-dot represent $G_{k}(s)$ for varying $k$. As $k$ increases, $G_{k}(s)$ approaches $G(s)$, which is represented by a solid line.

Verification of results: As discussed in section 2, relative modal controllabilities of swing modes can directly be compared by comparing the residues associated with corresponding modal signals (subject to the normalization criteria defined by Eqs. (6), (7) and (12). This concept is used to verify the results of the proposed scheme. Modal signal $\quad z_{m_{i}}=v_{i}^{T} z$ has modal observability $\gamma_{i}=v_{i}^{T} x_{i}=2 v_{\omega_{i}}^{T} x_{\omega_{i}}=1$. Thus, the modal signal can be synthesized using only the speed deviation measurements as

$$
\Delta \omega_{m_{i}}=2 v_{\omega_{i}}^{T} \Delta \omega
$$

which is essentially the modal speed signal that preserves the modal observability of $z_{m_{i}}$. A damping controller built using $\Delta \omega_{m i}$ as the feedback signal will be able to affect the $i$ th swing mode if at all it is controllable and vice-versa. Highly controllable swing modes will see higher movements as compared with less controllable swing modes for similar (and small) controller gains, when the controller is fed with the corresponding modal slip signals. The damping controller structure used for the case studies is as shown in figure 6. $T_{1}$ and $T_{2}$ are taken to be 1 for all the case studies; for a given value of $K_{d c}$, the controller provides almost similar gains at the swing mode frequencies.

In the verification procedure, the open-loop eigenvalues are first computed. Then with $\Delta \omega_{m i}$ as the feedback signal to the controller, the closed-loop eigenvalue corresponding to the $i$ th swing mode is evaluated and compared for the change in its value. This is done for all the 15 swing modes

Table 2. Partial list of the converged poles obtained using the proposed method; TCSC in the branch between buses 50 and 52 .

\begin{tabular}{|c|c|c|c|}
\hline Mode $\left(\lambda_{i}\right)$ & $\left|\rho_{i}\right|$ & $\left|\beta_{i}\right|=\sqrt{\left|\lambda_{i}\right|\left|\rho_{i}\right|}$ & Remarks \\
\hline$-0.0150 \pm j 3.5040$ & 2.9480 & 3.2140 & $\begin{array}{l}\text { Swing mode } \\
\text { G14 vs. G16 }\end{array}$ \\
\hline$-0.0960 \pm j 4.5470$ & 2.2000 & 3.1630 & $\begin{array}{c}\text { Swing mode } \\
\text { G10-G13 vs. G1,G9 }\end{array}$ \\
\hline$-0.0210 \pm j 2.6070$ & 3.6740 & 3.0950 & $\begin{array}{c}\text { Swing mode } \\
\text { G1-G13 vs. G14-G16 }\end{array}$ \\
\hline$-0.0450 \pm j 11.4420$ & 0.4020 & 2.1450 & $\begin{array}{l}\text { Swing mode } \\
\text { G10 vs. G11 }\end{array}$ \\
\hline$-0.0100 \pm j 5.0740$ & 0.8030 & 2.0180 & $\begin{array}{c}\text { Swing mode } \\
\text { G15 vs. G14,G16 }\end{array}$ \\
\hline $0.0970 \pm j 8.6050$ & 0.0370 & 0.5640 & $\begin{array}{c}\text { Swing mode } \\
\text { G1,G8 vs. G10,G11 }\end{array}$ \\
\hline$-0.0390 \pm j 7.4720$ & 0.0200 & 0.3860 & $\begin{array}{l}\text { Swing mode } \\
\text { G12 vs. G13 }\end{array}$ \\
\hline$-0.7750 \pm j 1.5130$ & 0.6980 & - & Non-swing mode \\
\hline$-0.8310 \pm j 1.1490$ & 0.2380 & - & Non-swing mode \\
\hline
\end{tabular}




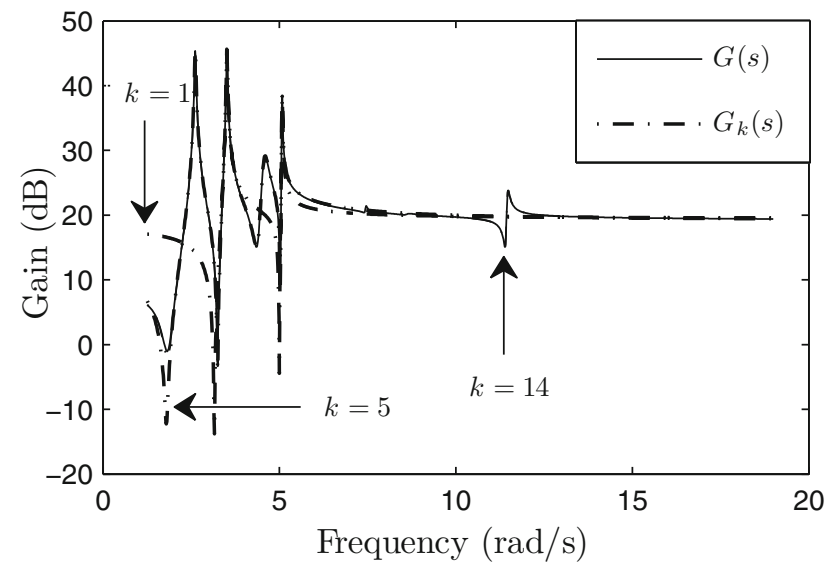

Figure 5. Bode magnitude plots for exact and reduced order transfer functions; TCSC in the branch between buses 50 and 52.

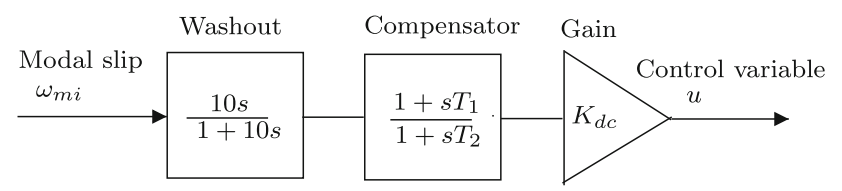

Figure 6. Modal slip-based damping controller.

of the system. The EIGS [21] function of MATLAB, which searches for a desired number of poles around a given shift point, is used (only) in the verification procedure. Note that, because of the moderate size of this test system, all the swing modes could be selectively traced out using EIGS.

Table 3 shows the movement of swing modes as $\left|K_{d c}\right|$ is varied from 0 to 0.05 with individual modal speed signals used (one at a time) as feedback signals in the controller. In each case, the polarity of $K_{d c}$ is determined from the transfer function residue associated with the corresponding modal speed. The following observations can be made.

(i) Swing modes (for example, poles $-0.0210 \pm j$ $2.6070,-0.0150 \pm j 3.5040$, etc.) that exhibit larger movements are in fact the high-residue $\left(\left|\rho_{i}\right|\right)$ modes to which the proposed algorithm has converged as shown in table 2.

(ii) Swing modes with low residues (for example, poles $0.0970 \pm j 8.6050,-0.0390 \pm j 7.4720$ and others to which the algorithm did not converge) have limited shifts or are practically unchanged. This validates the claim that highly controllable swing modes can be identified with relatively high residues in the transfer function between dual input-output pairs.

(iii) It can also be seen that the relative movements are roughly in proportion to the relative values of $\left|\beta_{i}\right|=$ $\sqrt{\left|\lambda_{i}\right|\left|\rho_{i}\right|}$ and therefore $\sqrt{\left|\lambda_{i}\right|\left|\rho_{i}\right|}$ can be used to approximately rank the swing modes for modal controllability.
Table 3. Swing mode movement due to corresponding modal slip; TCSC in the branch between buses 50 and 52; eigenvalues computed using EIGS.

\begin{tabular}{l|c|l}
\hline$\left|K_{d c}\right|=0.0$ & $\left|K_{d c}\right|=0.05$ & Remarks \\
\hline$-\mathbf{0 . 0 1 5 0} \pm j \mathbf{3 . 5 0 4 0}$ & $-\mathbf{0 . 1 9 0 0} \pm j \mathbf{3 . 4 9 2 0}$ & High modal \\
$-\mathbf{0 . 0 9 6 0} \pm j \mathbf{4 . 5 4 7 0}$ & $-\mathbf{0 . 2 9 1 0} \pm j \mathbf{4 . 5 7 1 0}$ & controllability \\
$-\mathbf{0 . 0 2 1 0} \pm j \mathbf{2 . 6 0 7 0}$ & $-\mathbf{0 . 1 6 5 0} \pm j \mathbf{2 . 6 1 6 0}$ & \\
$-\mathbf{0 . 0 4 5 0} \pm j \mathbf{1 1 . 4 4 2 0}$ & $-\mathbf{0 . 1 5 3 0} \pm j \mathbf{1 1 . 4 5 0 0}$ & \\
$\mathbf{- 0 . 0 1 0 0} \pm j \mathbf{5 . 0 7 4 0}$ & $-\mathbf{0 . 1 0 4 0} \pm j \mathbf{5 . 0 6 5 0}$ & \\
\hline $0.0970 \pm j 8.6050$ & $0.0710 \pm j 8.6050$ & Low modal \\
$-0.0390 \pm j 7.4720$ & $-0.0660 \pm j 7.4880$ & controllability \\
$-0.1190 \pm j 6.0060$ & $-0.1240 \pm j 6.0050$ & \\
$-0.2480 \pm j 6.7490$ & $-0.2530 \pm j 6.7520$ & \\
$-0.4060 \pm j 7.4260$ & $-0.4070 \pm j 7.4250$ & \\
$-0.3330 \pm j 7.8990$ & $-0.3340 \pm j 7.8990$ & \\
$-0.2390 \pm j 7.9770$ & $-0.2480 \pm j 7.9680$ & \\
$-0.4000 \pm j 9.4520$ & $-0.4030 \pm j 9.4520$ & \\
$-0.5880 \pm j 9.5430$ & $-0.5880 \pm j 9.5420$ & \\
$-0.6600 \pm j 9.3790$ & $-0.6600 \pm j 9.3800$ & \\
\hline
\end{tabular}

We repeat the case studies with a TCSC located between buses 13 and 14. Table 4 shows the controllable swing modes to which the process converges and table 5 validates the result.

Further insights regarding the results can be obtained by observing the mode shapes of the swing modes. While a FACTS device or HVDC link at a given location may have controllability over certain inter-area modes, and modes that are local to the areas near the device location, it is unlikely that it would exert control over modes that are local to far-off areas. This is observed in the obtained results. While for the TCSC located between buses 50 and 52 , controllability over local modes involving machines (G1-G9) in the far-off NETS area (e.g., $-0.2480 \pm j 6.7490,-0.3330 \pm j 7.8990)$ is negligible, for the TCSC located between buses 13 and 14, the local modes involving machines from the NETS system assume high controllability.

4.1b HVDC: The dc link power and reactive power exchange at converter buses are modelled as shunt current injections [22] at the terminal AC buses. Link power flow is considered as the controllable element, i.e., $u=\Delta P_{d c}$ and the difference between inverter and rectifier bus voltage phase angles is considered as the dual signal, i.e., $y_{d}=\phi_{d}$. We consider a HVDC link between buses 9 and 10. The voltage angle difference between buses 9 and 10 is considered as the dual signal. Table 6 shows a partial list of the converged poles. Table 7 validates the obtained results.

This location of the DC link exhibits good controllability over local modes of the NETS area besides being able to control inter-area modes involving machines from the 
Table 4. Partial list of the converged poles obtained using the proposed method; TCSC in the branch between buses 13 and 14 .

\begin{tabular}{|c|c|c|c|}
\hline Mode $\left(\lambda_{i}\right)$ & $\left|\rho_{i}\right|$ & $\left|\beta_{i}\right|=\sqrt{\left|\lambda_{i}\right|\left|\rho_{i}\right|}$ & Remarks \\
\hline$-0.2480 \pm j 6.7490$ & 8.2940 & 7.4840 & $\begin{array}{c}\text { Swing mode } \\
\text { G2,G3 vs. G4-G7 }\end{array}$ \\
\hline$-0.3330 \pm j 7.8990$ & 1.8040 & 3.7770 & $\begin{array}{l}\text { Swing mode } \\
\text { G2 vs. G3 }\end{array}$ \\
\hline$-0.2390 \pm j 7.9770$ & 0.8910 & 2.6670 & $\begin{array}{c}\text { Swing mode } \\
\text { G1,G8 vs. G2,G3,G6 }\end{array}$ \\
\hline$-0.0960 \pm j 4.5470$ & 0.3600 & 1.2800 & $\begin{array}{c}\text { Swing mode } \\
\text { G10-G13 vs. G1,G9 }\end{array}$ \\
\hline$-0.4060 \pm j 7.4260$ & 0.1460 & 1.0400 & $\begin{array}{c}\text { Swing mode } \\
\text { G4,G5 vs. G6,G7 }\end{array}$ \\
\hline$-0.1190 \pm j 6.0060$ & 0.0860 & 0.7190 & $\begin{array}{c}\text { Swing mode } \\
\text { G1,G8,G9 vs. G2-G7 }\end{array}$ \\
\hline
\end{tabular}

Table 5. Swing mode movement due to corresponding modal slip; TCSC in the branch between buses 13 and 14; eigenvalues computed using EIGS.

\begin{tabular}{l|c|l}
\hline$\left|K_{d c}\right|=0.0$ & $\left|K_{d c}\right|=0.025$ & Remarks \\
\hline $\mathbf{- 0 . 2 4 8 0} \pm j \mathbf{6 . 7 4 9 0}$ & $\mathbf{- 0 . 4 3 8 0} \pm j \mathbf{6 . 7 5 1 0}$ & High modal \\
$-\mathbf{0 . 3 3 3 0} \pm j \mathbf{7 . 8 9 9 0}$ & $-\mathbf{0 . 4 2 0 0} \pm j \mathbf{7 . 8 9 7 0}$ & controllability \\
$-\mathbf{0 . 2 3 9 0} \pm j \mathbf{7 . 9 7 7 0}$ & $-\mathbf{0 . 2 8 5 0} \pm j \mathbf{7 . 9 5 4 0}$ & \\
\hline$-0.0960 \pm j 4.5470$ & $-0.1220 \pm j 4.5430$ & Low modal \\
$-0.4060 \pm j 7.4260$ & $-0.4320 \pm j 7.4340$ & controllability \\
$-0.1190 \pm j 6.0060$ & $-0.1380 \pm j 6.0050$ & \\
$-0.0150 \pm j 3.5040$ & $-0.0170 \pm j 3.5040$ & \\
$-0.0210 \pm j 2.6070$ & $-0.0390 \pm j 2.6090$ & \\
$-0.0100 \pm j 5.0740$ & $-0.0110 \pm j 5.0740$ & \\
$0.0970 \pm j 8.6050$ & $0.0910 \pm j 8.5950$ & \\
$-0.0390 \pm j 7.4720$ & $-0.0450 \pm j 7.4780$ & \\
$-0.4000 \pm j 9.4520$ & $-0.4120 \pm j 9.4610$ & \\
$-0.5880 \pm j 9.5430$ & $-0.6100 \pm j 9.5410$ & \\
$-0.6600 \pm j 9.3790$ & $-0.6770 \pm j 9.3910$ & \\
$-0.0450 \pm j 11.4420$ & $-0.0470 \pm j 11.4420$ & \\
\hline
\end{tabular}

NETS and NYPS. However, the controllability over modes that are local to the far-off external area (e.g., $-0.0150 \pm$ $j 3.5050$ and $-0.0100 \pm j 5.0750)$ is not high.

\subsection{Indian power grid}

The Indian power system is a large interconnected system consisting of five subsystems, namely the Northern (NR), Eastern(ER), North-eastern(NER), Western (WR) and Southern (SR) regions. Network and powerflow data for this system are obtained from the POC data of the year 2014-15 available in [23]. Typical dynamic data for generators based on their MW rating and type are taken from [24]. The generators are assumed to be equipped with either a static or a brush-less exciter for which the typical model parameters are obtained from [25]. Loads are modelled as composite ZIP loads with static voltage and frequency dependence. In buses where the quiescent real power component of loads exceed $100 \mathrm{MW}, 25 \%$ of the loads are modelled as dynamic induction machine loads. The system considered here has 7242 buses, 8729 transmission lines, 3745 transformers 1232 generators, 5 TCSC compensated lines, 9 embedded HVDC links and 1 SVC.

We consider the HVDC link that joins Talcher in the ER to Kolar in SR. The location of the HVDC link in the Indian system can be understood from figure 7. This is a bipolar HVDC link that operates at a DC voltage of $\pm 500 \mathrm{kV}$ and has a quiescent power flow of $2000 \mathrm{MW}$. The converter at

Table 6. Partial list of the converged poles obtained using the proposed method; HVDC link between buses 9 and 10 .

\begin{tabular}{|c|c|c|c|}
\hline Mode $\left(\lambda_{i}\right)$ & $\left|\rho_{i}\right|$ & $\left|\beta_{i}\right|=\sqrt{\left|\lambda_{i}\right|\left|\rho_{i}\right|}$ & Remarks \\
\hline$-0.0940 \pm j 4.5320$ & 0.0230 & 0.3210 & $\begin{array}{c}\text { Swing mode } \\
\text { G10-G13 vs. G1,G9 }\end{array}$ \\
\hline$-0.2470 \pm j 6.7380$ & 0.0150 & 0.3170 & $\begin{array}{c}\text { Swing mode } \\
\text { G2,G3 vs. G4-G7 }\end{array}$ \\
\hline$-0.0220 \pm j 2.5960$ & 0.0170 & 0.2130 & $\begin{array}{c}\text { Swing mode } \\
\text { G1-G13 vs. G14-G16 }\end{array}$ \\
\hline$-0.1190 \pm j 6.0060$ & 0.0030 & 0.1360 & $\begin{array}{l}\text { Swing mode } \\
\text { G9 vs. G2-G7 }\end{array}$ \\
\hline$-0.3320 \pm j 7.8840$ & 0.0020 & 0.1230 & $\begin{array}{l}\text { Swing mode } \\
\text { G2 vs. G3 }\end{array}$ \\
\hline$-0.830 \pm j 1.152$ & 0.0040 & - & Non-swing mode \\
\hline$-1.647 \pm j 0.055$ & 0.0021 & - & Non-swing mode \\
\hline
\end{tabular}


Table 7. Swing mode movement due to corresponding modal slip; HVDC link between buses 9 and 10; eigenvalues computed using EIGS.

\begin{tabular}{l|c|l}
\hline$\left|K_{d c}\right|=0.0$ & $\left|K_{d c}\right|=0.5$ & Remarks \\
\hline $\mathbf{- 0 . 0 9 4 0} \pm j \mathbf{4 . 5 3 2 0}$ & $-\mathbf{0 . 2 7 1 0} \pm j \mathbf{4 . 5 7 8 0}$ & High modal \\
$-\mathbf{0 . 2 4 7 0} \pm j \mathbf{6 . 7 3 8 0}$ & $-\mathbf{0 . 4 4 8 0} \pm j \mathbf{6 . 7 6 0 0}$ & controllability \\
$-\mathbf{0 . 0 2 2 0} \pm j \mathbf{2 . 5 9 6 0}$ & $-\mathbf{0 . 2 4 7 0} \pm j \mathbf{2 . 5 8 6 0}$ & \\
$-\mathbf{0 . 1 1 9 0} \pm j \mathbf{6 . 0 0 6 0}$ & $-\mathbf{0 . 1 9 5 0} \pm j \mathbf{6 . 0 0 2 0}$ & \\
$-\mathbf{0 . 3 3 2 0} \pm j \mathbf{7 . 8 8 4 0}$ & $-\mathbf{0 . 4 0 5 0} \pm j \mathbf{7 . 8 8 9 0}$ & \\
\hline$-0.2390 \pm j 7.9750$ & $-0.2840 \pm j 7.9650$ & Low modal \\
$-0.0150 \pm j 3.5050$ & $-0.0340 \pm j 3.5040$ & controllability \\
$-0.0100 \pm j 5.0750$ & $-0.0190 \pm j 5.0740$ & \\
$-0.0430 \pm j 7.4610$ & $-0.0590 \pm j 7.4540$ & \\
$-0.4060 \pm j 7.4260$ & $-0.4160 \pm j 7.4330$ & \\
$-0.3980 \pm j 9.4510$ & $-0.4030 \pm j 9.4430$ & \\
$-0.5870 \pm j 9.5410$ & $-0.5960 \pm j 9.5420$ & \\
$-0.6590 \pm j 9.3790$ & $-0.6680 \pm j 9.3860$ & \\
$0.0938 \pm j 8.6056$ & $0.0520 \pm j 8.6440$ & \\
$-0.0460 \pm j 11.4480$ & $-0.0560 \pm j 11.4660$ & \\
\hline
\end{tabular}

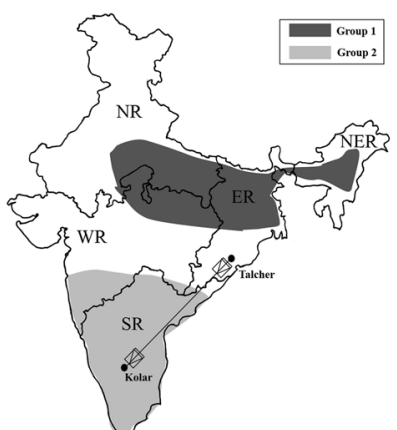

(a)

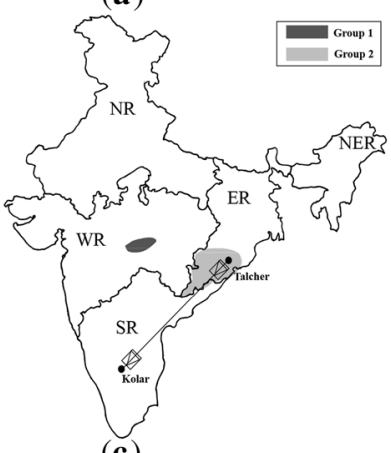

(c)
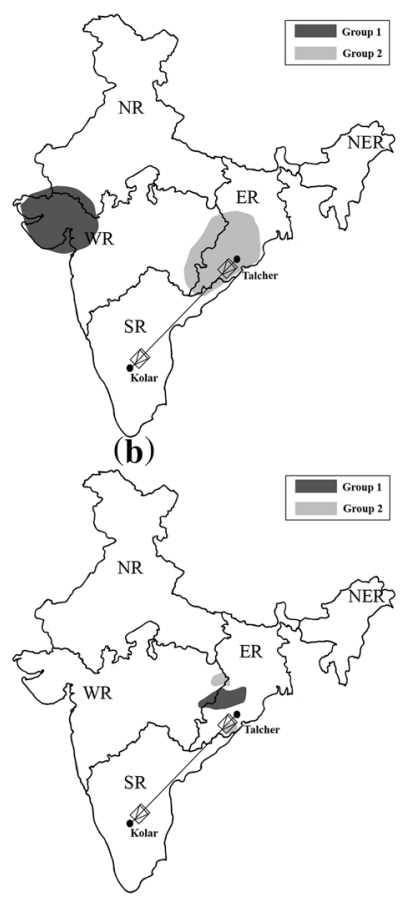

(d)
Figure 7. Coherent generator (with high participation) groups across highly controllable swing modes: (a) $-0.2350 \pm j 1.3190$; (b) $\quad-0.1340 \pm j 4.4870 ; \quad$ (c) $-0.1530 \pm j 5.9300$;
$-0.7250 \pm j 11.5460$.

Talcher is the rectifier, while that at Kolar is the inverter end of the link. Considering the power flow on the DC link as the controllable parameter, the bus voltage angular difference between Talcher bus and Kolar bus is taken as the dual signal. For the computations, $\hat{n}$ is set to 10 . The

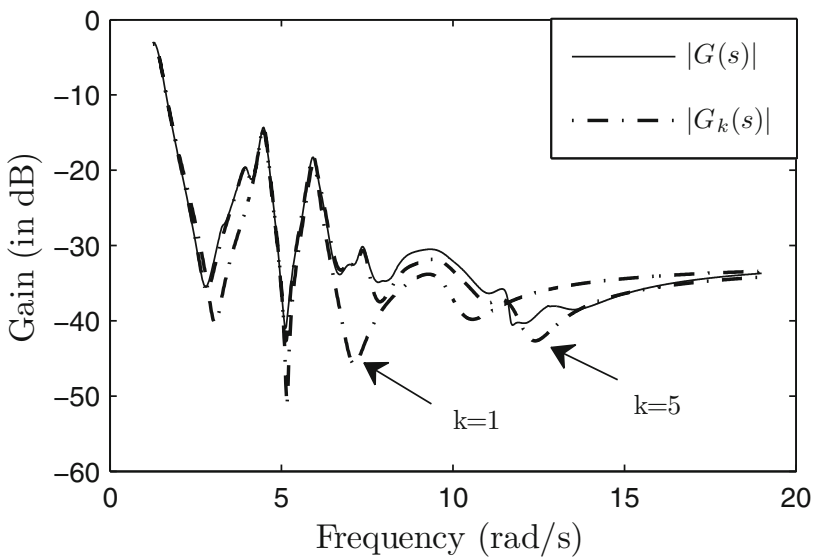

Figure 8. Bode magnitude plots for exact and reduced order transfer functions; HVDC link between Talcher and Kolar.

Table 8. Partial list of the converged poles obtained using the proposed method; HVDC link between Talcher and Kolar.

\begin{tabular}{llcc}
\hline Mode $\left(\lambda_{i}\right)$ & $\left|\rho_{i}\right|$ & $\left|\beta_{i}\right|=\sqrt{\left|\lambda_{i}\right|\left|\rho_{i}\right|}$ & Remarks \\
\hline$-0.2350 \pm j 1.3190$ & 0.16 & 0.463 & Swing mode \\
$-0.7250 \pm j 11.5460$ & 0.011 & 0.3568 & Swing mode \\
$-0.1340 \pm j 4.4870$ & 0.024 & 0.3282 & Swing mode \\
$-0.7920 \pm j 9.6750$ & 0.011 & 0.3268 & Swing mode \\
$-0.1530 \pm j 5.9300$ & 0.017 & 0.3175 & Swing mode \\
$-0.9040 \pm j 11.6110$ & 0.006 & 0.2644 & Swing mode \\
$-0.7980 \pm j 10.6950$ & 0.006 & 0.2536 & Swing mode \\
$-0.1740 \pm j 3.9800$ & 0.01 & 0.1995 & Swing mode \\
$-1.3380 \pm j 0.0240$ & 0.007 & - & Non-swing \\
& & & mode \\
$-1.1300 \pm j 0.3190$ & 0.006 & - & Non-swing \\
& & & mode \\
\hline
\end{tabular}

computation is carried out till the transfer function convergence error $e_{k}^{r}$ reduces below 0.05 .

The transfer function convergence is illustrated in figure 8 . A total of 50 modes are found, out of which 35 are identified as swing modes. A partial list of the converged eigenvalues is shown in table 8. Examination of mode shapes and slip participation for the $-0.2346 \pm j 1.3189 \mathrm{rad} / \mathrm{s}$ mode shows that it is the inter-area mode between machines in northern and southern regions. The same is depicted in figure 7a. The coherent group of machines in NR that oscillate against the coherent group of machines in SR are shown in different shades. This is intuitive as the embedded DC link that spans from the northern to the southern half of the system is expected to exhibit high controllability over the north-south inter-area mode. Similar examination of mode shapes for a few other controllable modes have been done and the groups of coherent machines across each of them are shown in figure $7 \mathrm{~b}-\mathrm{d}$. The mode corresponding to eigenvalues $-0.1340 \pm j 4.4870$ is found to be an inter-area mode between ER and WR. Modes corresponding to 
eigenvalues $-0.1530 \pm j 5.9300$ and $-0.7250 \pm j 11.5460$ are swing modes local to the converter location at Talcher.

Dimension of system matrices $A$ and $E$ is $31903 \times 31903$. The total computational time is approximately $420 \mathrm{~s}$. This includes the time elapsed in calculation of the actual frequency response ${ }^{2} G(s)$ (approximately $240 \mathrm{~s}$ ) and evaluation of the controllable swing modes (approximately $180 \mathrm{~s}$ ) as per the steps shown in figure 3 . The fast and effective computation can be attributed to the subspace iteration and deflation schemes associated with SADPA.

\section{Conclusion}

The paper presents a method to compute the controllable swing mode spectrum of FACTS/HVDC-based damping controllers in large power systems. The aim of the proposed scheme is to find the controllable subset of the swing modes without any reference to a particular controller and feedback signal. Appropriate normalization of eigenvectors and choice of feedback signals allow mapping of the problem of finding the swing modes with high modal controllability to that of the swing modes that have high transfer function residues. With this mapping, we have leveraged the efficient SADPA algorithm as the engine for an iterative process for the computation of the controllable swing modes. This is illustrated by case studies with a moderately sized IEEE 16-machine 68-bus system and a practical large power system (Indian power system).

The efficacy of the approach and reliability of the results are established by verification with modal signal (as the feedback signal)-based damping controllers. A modal signal guarantees observability of a mode. Therefore, insensitivity of a mode to the modal signal-based damping controller implies limited modal controllability. This concept was used to verify the obtained results. Numerical results with the Indian power system illustrate the "computational time" for the practical systems with a large number of states. Specifically for the case study in this paper, the Talcher-Kolar HVDC link is chosen. The NorthSouth inter-area mode is found to be the most highly controllable swing mode. The East-West inter-area mode is also well controlled by this HVDC link. As can be expected, the link also exhibits good modal controllability over a few modes that are local to the DC link.

\section{Acknowledgements}

The authors gratefully acknowledge the contributions of $\mathrm{Mr}$ Abishek R S and Mr A Sinkar in preparation of the small

${ }^{2} G(s)$ is calculated using Eq. (16) for a frequency range of $1.26-19 \mathrm{rad} / \mathrm{s}$ in steps of $0.01 \mathrm{rad} / \mathrm{s}$. signal model of the Indian Power System. Both of them have received their Master's degrees from the Department of Electrical Engineering, IIT Bombay.

\section{Appendix I. Derivation of constraint in Eq. (12)}

Following a perturbation, the energy in the disturbance can be expressed as sum of potential and kinetic energy as follows:

$$
\begin{aligned}
E & =\frac{1}{2}\left(\Delta \delta^{T} A_{r} \Delta \delta+\Delta \omega^{T} M \Delta \omega\right) \\
& =\frac{1}{2}\left[\begin{array}{c}
\Delta \delta \\
\Delta \omega
\end{array}\right]^{T}\left[\begin{array}{ll}
A_{r} & {[0]} \\
{[0]} & M
\end{array}\right]\left[\begin{array}{c}
\Delta \delta \\
\Delta \omega
\end{array}\right] .
\end{aligned}
$$

It is constant for the unforced system (i.e., $u=0$ ). If the $i$ th mode only is excited, then from Eq. (5) we have

$$
\left[\begin{array}{c}
\Delta \delta \\
\Delta \omega
\end{array}\right]=\left[\begin{array}{c}
x_{\delta_{i}} \\
x_{\omega_{i}}
\end{array}\right] z_{m_{i}}+\left[\begin{array}{c}
x_{\delta_{i}}^{*} \\
x_{\omega_{i}}^{*}
\end{array}\right] z_{m_{i}}^{*} .
$$

Using Eq. (A2) in Eq. (A1), it can be shown that

$$
\begin{aligned}
E=\frac{1}{2}\left[z_{m_{i}}^{2}\left(x_{\delta_{i}}^{T} A_{r} x_{\delta_{i}}+x_{\omega_{i}}^{T} M x_{\omega_{i}}\right)+\left(z_{m_{i}}^{*}\right)^{2}\left(x_{\delta_{i}}^{H} A_{r} x_{\delta_{i}}^{*}+x_{\omega_{i}}^{H} M x_{\omega_{i}}^{*}\right)\right. \\
\left.+2\left|z_{m_{i}}\right|^{2}\left(x_{\delta_{i}}^{H} A_{r} x_{\delta_{i}}+x_{\omega_{i}}^{H} M x_{\omega_{i}}\right)\right] .
\end{aligned}
$$

From Eqs. (3) and (4), it can be shown that

$$
\begin{aligned}
& x_{\delta_{i}}^{T} A_{r} x_{\delta_{i}}+x_{\omega_{i}}^{T} M x_{\omega_{i}}=0, \\
& x_{\delta_{i}}^{H} t A_{r} x_{\delta_{i}}^{*}+x_{\omega_{i}}^{H} M x_{\omega_{i}}^{*}=0,
\end{aligned}
$$

and therefore

$$
E=\left|z_{m_{i}}\right|^{2}\left(x_{\delta_{i}}^{H} A_{r} x_{\delta_{i}}+x_{\omega_{i}}^{H} M x_{\omega_{i}}\right)=E_{i}
$$

where $E_{i}$ denotes the energy when only the $i$ th mode is excited. It can be shown that when several modes are excited, the disturbance energy is given by the sum of all modal energies, i.e, $E=\sum_{i=1}^{\left(n_{g}-1\right)} E_{i}$. Note that $E_{i}$ is also a constant and does not depend on scaling of the eigenvectors.

The strength of the modal signal $y=v_{i}^{T} z=z_{m_{i}}$ is given by its amplitude, which is equal to $\left|z_{m_{i}}\right|$. When only one mode is excited at a time, with the same disturbance energy $E_{i}$, strength of the modal signal being equal across modes implies $\left(x_{\delta_{i}}^{H} A_{r} x_{\delta_{i}}+x_{\omega_{i}}^{H} M x_{\omega_{i}}\right)$ is the same across all modes (as seen from Eq. (A6)). Without loss of generality, we assign its value to be 1 and obtain the following condition:

$$
x_{\delta_{i}}^{H} A_{r} x_{\delta_{i}}+x_{\omega_{i}}^{H} M x_{\omega_{i}}=1 .
$$


Using the initial assumption of $x_{\delta_{i}}$ being real, we obtain the result of Eq. (12).

\section{Appendix II. Example of dominant pole at infinity}

Let us consider the state-space matrices $E=\left[\begin{array}{ll}I & 0 \\ 0 & 0\end{array}\right], A=\left[\begin{array}{ll}\alpha & 0 \\ 0 & \alpha\end{array}\right]$ where $\alpha=\left[\begin{array}{cc}0.1 & 0.2 \\ -0.2 & 0.3\end{array}\right]$ and $b=c=\left[\begin{array}{lllll}0.1 & 0.1 & 1.0 & 1.0\end{array}\right]^{T}$. Generalized eigenvalue analysis of this system yields a mode $0.2 \pm 0.17321 i$ with residue of 0.01 and two poles at infinity with residue of $\infty$.

Trajectories of the eigenvalue estimate over iterations, when SADPA is applied on this system with $\left|\rho_{i}\right|$ (or $\left|\rho_{i}\right|\left|\lambda_{i}\right|$ ) (case (a)), and $\left|\rho_{i}\right| /\left|\lambda_{i}\right|$ (case (b)) as selection criteria separately are shown in the following table. It is clearly seen that the selection criterion in case (b) avoids convergence to the dominant pole at $\infty$.

\begin{tabular}{ccc}
\hline & \multicolumn{2}{c}{ Eigen-estimate } \\
\cline { 2 - 3 } Iteration & Case (a) & Case (b) \\
\hline 0 & $0+j 0.1700$ & $0+j 0.1700$ \\
1 & $25.2768-j 5.1847$ & $25.2768-j 5.1847$ \\
2 & $(2.72+j 3.50) \times 10^{5}$ & $0.2944+j 0.1997$ \\
3 & $\infty$ & $0.2000-j 0.1732$ \\
\hline
\end{tabular}

\section{List of symbols}

$\begin{array}{ll}A, b, c, d & \text { state-space matrices } \\ z & \text { state variables } \\ \delta, \omega & \text { rotor angle (rad), speed (rad/s) } \\ n_{g} & \text { number of generators } \\ \Omega_{i} & \text { frequency of the } i \text { th swing mode (rad/s) } \\ x_{\delta_{i}}, x_{\omega_{i}} & \text { components of right eigenvector corresponding } \\ & \text { to rotor angle and speed, respectively } \\ v_{\delta_{i}}, v_{\omega_{i}} & \text { components of left eigenvector corresponding } \\ & \text { to rotor angle and speed, respectively } \\ \operatorname{Re}(.) & \text { real part of a complex number } \\ (.)^{*} & \text { element-wise conjugate of a complex vector } \\ (.)^{T} & \text { transpose } \\ (.)^{H} & \text { conjugate-transpose of a vector } \\ G(s) & \text { SISO transfer function } \\ \mathrm{SSPF} & \text { sum of slip participation factors }\end{array}$

\section{References}

[1] Padiyar K R 2007 FACTS controllers in power transmission and distribution. New Delhi: New Age International(P) Ltd

[2] Padiyar K R 2012 HVDC power transmission systems, 2nd ed. New Delhi: New Age International(P) Ltd
[3] Martins N, Lima L T G and Pinto H J C P 1996 Computing dominant poles of power system transfer functions. IEEE Trans. Power Syst. 11(1): 162-167

[4] Uchida N and Nagao T 1988 A new eigen-analysis method of steady-state stability studies for large power systems: Smatrix method. IEEE Trans. Power Syst. 3(2): 2085-2092

[5] Semlyen A and Wang L 1988 Sequential computation of the complete eigen system for the study zone in small signal stability analysis of large power systems. IEEE Trans. Power Syst. 3(2): 715-725

[6] Wang L and Semlyen A 1989 Application of sparse eigenvalue techniques to the small signal stability analysis of large power systems. In: Proceedings of the Power Industry Computer Application Conference, pp. 358-365

[7] Angelidis G and Semlyen A 1995 Efficient calculation of critical eigenvalue clusters in the small signal stability analysis of large power systems. IEEE Trans. Power Syst. 10(1): 427-432

[8] Yang D and Ajjarapu V 2007 Critical eigenvalues tracing for power system analysis via continuation of invariant subspaces and projected Arnoldi method. IEEE Trans. Power Syst. 22(1): 324-332

[9] Li Y, Geng G and Jiang Q 2016 An efficient parallel KrylovSchur method for eigen-analysis of large-scale power system. IEEE Trans. Power Syst. 31(2): 920-930

[10] Rommes J and Martins N 2008 Computing large-scale system eigenvalues most sensitive to parameter changes, with applications to power system small-signal stability. IEEE Trans. Power Syst. 23(2): 434-442

[11] Chung C Y and Dai B 2013 A combined TSA-SPA algorithm for computing most sensitive eigenvalues in large-scale power systems. IEEE Trans. Power Syst. 28(1): 149-157

[12] Chung C Y and Dai B 2015 A generalized approach for computing most sensitive eigenvalues with respect to system parameter changes in large-scale power systems. IEEE Trans. Power Syst. pp. 1-11, https://doi.org/10.1109/ TPWRS.2015.2445792

[13] Byerly R T, Bennon R J and Sherman D E 1982 Eigenvalue analysis of synchronizing power flow oscillations in large electric-power systems. IEEE Trans. Power Appl. Syst. PAS101: 235-243

[14] Wong D Y, Rogers G J, Porretta B and Kundur P 1988 Eigenvalue analysis of very large power systems. IEEE Trans. Power Syst. 3(2): 472-480

[15] Martins N 1997 The dominant pole spectrum eigensolver. IEEE Trans. Power Syst. 12(1): 245-254

[16] Rommes J and Martins N 2006 Efficient computation of transfer function dominant poles using subspace acceleration. IEEE Trans. Power Syst. 21(3): 1471-1483

[17] Mhaskar U P and Kulkarni A M 2006 Power oscillation damping using FACTS devices: modal controllability, observability in local signals, and location of transfer function zeros. IEEE Trans. Power Syst. 21(1): 285-294

[18] Rogers G 2000 Power system oscillations. Norwell, MA: Kluwer

[19] Rommes J 2007 Methods for eigenvalue problems with applications in model order reduction. $\mathrm{PhD}$ Thesis, Utrecht University, Utrecht, http://dspace.library.uu.nl/handle/1874/ 21787 [Accessed 28 October 2016]

[20] Shubhanga K N and Anatholla Y 2000 Manual for a multimachine small-signal stability programme (Version 1.0). 
Department of Electrical Engineering, NITK Surathkal, Karnataka, India, p 54

[21] EIGS. http://www.mathworks.com/help/matlab/ref/eigs.html [Accessed 28 October 2016]

[22] Latorre H F, Ghandhari M and Soder L 2008 Active and reactive power control of VSC-HVDC. Electr. Power Syst. Res. 78: 1756-1763
[23] Power System Operation Corporation Ltd. POC data, https:// posoco.in/transmission-pricing/poc-data [Accessed 28 October 2016]

[24] Central Electricity Authority manual on transmission line planning criteria, http://cea.nic.in [Accessed 28 October 2016]

[25] Kundur P 1994 Power systems stability and control. New York: McGraw-Hill 